\title{
Elevated plasma fibrinogen level shows superior prognostic value than Epstein-Barr virus DNA load for stage IVA/B nasopharyngeal carcinoma patients in the intensity-modulated radiotherapy era
}

\author{
Mei Lan ${ }^{1, *}$, Chunyan Chen ${ }^{1, *}$, Ying Huang ${ }^{1}$, Minjie Mao $^{2}$, Fei Han ${ }^{1}$, Junfang Liao ${ }^{1}$, \\ Meiling Deng ${ }^{1}$, Zhijun Duan ${ }^{4}$, Lie Zheng ${ }^{3}$, Shaoxiong Wu ${ }^{1}$, Taixiang Lu ${ }^{1}$, Yutao Jian ${ }^{5}$ \\ ${ }^{1}$ Department of Radiation Oncology, State Key Laboratory of Oncology in Southern China, Sun Yat-sen University Cancer \\ Center, Collaborative Innovation Center for Cancer Medicine, Guangzhou, China \\ ${ }^{2}$ Department of Clinical Laboratory, State Key Laboratory of Oncology in Southern China, Sun Yat-sen University Cancer \\ Center, Collaborative Innovation Center for Cancer Medicine, Guangzhou, China \\ ${ }^{3}$ Department of Imaging Diagnosis and Interventional Center, State Key Laboratory of Oncology in Southern China, Sun Yat- \\ sen University Cancer Center, Collaborative Innovation Center for Cancer Medicine, Guangzhou, China \\ ${ }^{4}$ Department of Radiation Diagnosis and Interventional Center, Chengdu Military General Hospital, Chengdu, China \\ ${ }^{5}$ Institute of Stomatological Research, Sun Yat-sen University, Guangdong Provincial Key Laboratory of Stomatology, \\ Guangzhou, China \\ "These authors have contributed equally to this work \\ Correspondence to: Shaoxiong Wu, email: wushx@sysucc.org.cn \\ Taixiang Lu, email: lutx@sysucc.org.cn \\ Yutao Jian, email: jianyt@mail.sysu.edu.cn
}

Keywords: fibrinogen, nasopharyngeal carcinoma, stage IVA/B disease, Epstein-Barr viral DNA, prognosis

Received: March 30, 2016

Accepted: May 29, 2016

Published: June 15, 2016

\section{ABSTRACT}

Purpose: Effective prognostic factors for patients with stage IVA/B nasopharyngeal carcinoma (NPC) who are susceptible to distant metastases are limited. We aim to investigate the prognostic value of pretreatment plasma fibrinogen (FIB) level and Epstein-Barr virus DNA (EBV-DNA) load in these patients in the era of intensity-modulated radiotherapy (IMRT).

Results: The 5-year DSS, DFS and DMFS rates of the entire cohort were $72.7 \%$, $66.8 \%, 80.0 \%$, respectively. High FIB level was identified as a negative prognostic factor for survival: the 5-year DSS, DFS and DMFS rates for patients with high FIB (> $4.0 \mathrm{~g} / \mathrm{L}$ ) and normal FIB ( $\leq 4.0 \mathrm{~g} / \mathrm{L}$ ) were $60.3 \%$ vs. $76.0 \%, 56.0 \%$ vs. $69.9 \%$, and $59.4 \%$ vs. $85.5 \%$, respectively (all $P<0.001$ ). Subgroup analysis demonstrated that DSS, DFS and DMFS decreased as FIB gradually increased, even within the normal range. The risk of distant metastasis in patients with high FIB was over 3-fold than patients with normal FIB. EBV-DNA was not an independent prognostic factor for any survival outcomes in multivariate analysis.

Conclusion: High pretreatment FIB level shows superior prognostic value than EBV-DNA load for stage IVA/B NPC patients in the era of IMRT.

Materials and Methods: A total of 755 patients with newly-diagnosed stage IVA/B NPC treated with definitive IMRT between January 2007 and December 2011 were enrolled. Plasma FIB and EBV-DNA were measured before treatment. Disease-specific survival (DSS), disease-free survival (DFS) and distant metastasis-free survival (DMFS) were calculated using the Kaplan-Meier method; differences were compared using the log-rank test. 


\section{INTRODUCTION}

Nasopharyngeal carcinoma (NPC), a malignant tumor type with a distinct geographical and ethnic pattern of incidence, is most common in southern China. Due to its atypical symptoms and concealed location, almost one third of patients are diagnosed with stage IVA/B disease. The prognosis of stage IVA/B disease is much poorer than stage III disease and the 5-year overall survival rate is still around $60 \%$ to $70 \%$, even though intensity-modulated radiotherapy (IMRT) has been widely used. [1-3] Therefore, it is important to identify effective prognostic factors for this subgroup of patients.

Coagulation and activation of the fibrinolytic system are frequently observed in patients with cancer, and are associated with higher risks of progression, metastasis and poor outcome. [4-6] A relationship between cancer, hemostasis and inflammation has been widely accepted and over-expression of a number of procoagulant and fibrinolytic factors, especially fibrinogen (FIB), has been reported in malignant tumors. $[4,5]$ Extensive studies indicate a specific correlation between high plasma FIB level and the progressive and metastatic behaviour of tumour cells. $[7,8]$ An elevated pretreatment plasma FIB level has been identified as a significant negative prognostic factor for poorer survival in various solid tumour types, including lung, breast, oesophageal cancers and NPC. [6, 9-14] However, for NPC patients, findings from previous study [6] were based on stage I-IV diseases and different radiotherapy techniques. The role of pretreatment FIB level in stage IVA/B NPC patients treated with IMRT remains unknown.

Although pretreatment Epstein-Barr virus DNA (EBV-DNA) load has been regarded as a useful diagnostic biomarker for NPC for many years [15-18]. However, in our clinical experience, unlike many patients who have stage III diseases, patients with stage IVA/B diseases usually have extremely high EBV-DNA loads. Whether elevated EBVDNA remains to be an effective prognostic biomarker for stage IVA/B patients deserves further investigation.

Therefore, we conducted a retrospective study of a large cohort of patients to investigate the prognostic value of pretreatment plasma FIB level and EBV-DNA loads in stage IVA/B NPC in the era of IMRT.

\section{RESULTS}

\section{Survival outcomes of patients with different pretreatment plasma FIB levels}

Within a median follow-up period of 50 months (range, 3-87 months), 194/755 (25.7\%) patients died and 236/755 (31.3\%) patients developed disease progression. One fifth of patients $(20.1 \%, 152 / 755)$ had high plasma FIB (> $4.0 \mathrm{~g} / \mathrm{L})$ levels, of whom $61 / 152(40.1 \%)$ died and $68 / 152(44.7 \%)$ suffered treatment failure. The 5-year DSS, DFS and DMFS rates for all patients were $72.7 \%$,
$66.8 \%, 80.0 \%$, respectively. The rates of 5-year DSS, DFS and DMFS for patients with high FIB levels were significantly poorer than those of patients with normal FIB levels $(60.3 \%$ and $76.0 \%, 56.0 \%$ vs. $69.9 \%, 59.4 \%$ and $85.5 \%$, respectively; all $P<0.001$; Figure 1).

Patients with stage IVA NPC had a better prognosis than patients with stage IVB NPC, with 5-year DSS, DFS and DMFS rates of $74.7 \%$ vs. $69.2 \%(P=0.042), 69.3 \%$ vs. $62.5 \%(P=0.022)$, and $82.1 \%$ vs. $76.0 \%(P=0.009)$, respectively. Survival analysis of the patients with stage IVA NPC showed that high FIB level was associated with significantly poorer DSS, DFS and DMFS than normal FIB level (all $P<0.001$ ). High FIB level was also associated with poorer DMFS $(P<0.001)$, but not DSS or DFS, compared to normal FIB in patients with stage IVB NPC (Table 1).

To further assess the prognostic value of FIB, we divided all patients into four equally-sized subgroups using the upper quartile, median, and lower quartile FIB levels, as follows: $<2.8 \mathrm{~g} / \mathrm{L}, 188$ cases (Group 1); 2.8-3.6 g/L, 194 cases (Group 2), 3.7-4.6 g/L, 189 cases (Group 3); > $4.6 \mathrm{~g} / \mathrm{L}, 184$ cases (Group 4). Survival curves for the four subgroups are shown in Figure 2. Significant associations were observed between increased FIB and reduced DSS, DFS and DMFS (all $P<0.05$ ). Small increases in plasma FIB, even within the normal range, had a significant negative prognostic impact on survival, which indicates that gradual increases in pretreatment plasma FIB are associated with poorer survival outcomes.

\section{Treatment outcomes for patients with different pretreatment EBV-DNA loads}

The median EBV-DNA load was 10000 copies/ $\mathrm{ml}$ (range, 0-10600000 copies/ml). The 5-year DFS and DMFS rates of patients with an EBV-DNA load $>10000$ copies $/ \mathrm{ml}$ were inferior to those of patients with an EBVDNA load $\leq 10000$ copies $/ \mathrm{ml}(62.0 \%$ vs. $71.4 \%, 75.8 \%$ vs. $84.2 \%$, respectively, $P<0.02)$; however, no significant difference in DSS was observed between patients with high and low EBV-DNA loads $(P=0.066)$. (Figure 3 )

To further evaluate the prognostic value of EBVDNA, we then divided all patients into four equally-sized subgroups according to the upper quartile, median, and lower quartile EBV-DNA loads, as follows: $<1000$ copies/ ml, 199 cases (Group A); 1000-9999 copies/ml, 188 cases (Group B); 10000-50000 copies/ml, 186 cases (Group C), $>50000,182$ cases (Group D). DMFS slightly reduced as the EBV-DNA load increased, and the survival curves were very close to each other (Figure 4); however, EBVDNA load was not significantly associated with DSS or DFS (all $P$ values $>0.05$ ).

\section{Univariate and multivariate analyses}

Univariate analysis of all 755 patients indicated that FIB, EBV-DNA, $\mathrm{N}$ category, clinical stage, age 

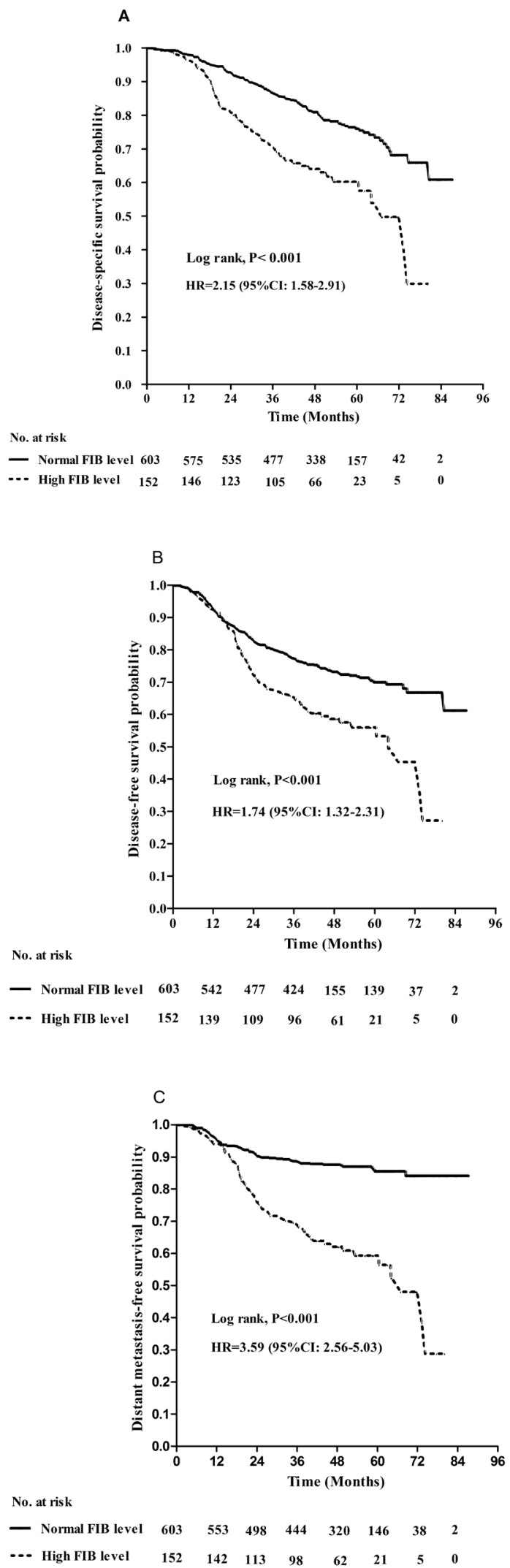

Figure 1: Kaplan-Meier A. disease-specific survival, B. disease-free survival and C. distant metastasis-free survival curves for patients with stage IVA/B NPC stratified by pretreatment plasma FIB $(>4 \mathrm{~g} / \mathrm{L}$ vs. $\leq 4 \mathrm{~g} / \mathrm{L}) . P$-values were calculated using the unadjusted log-rank test; Hazards ratios (HR) and 95\% confidence intervals (CIs) were calculated using the unadjusted Cox proportional hazards model. 
Table 1: Survival analysis of patients with stage IVA or IVB nasopharyngeal carcinoma stratified by different pretreatment plasma FIB levels

\begin{tabular}{|c|c|c|c|c|c|c|}
\hline & \multicolumn{2}{|c|}{ Stage IVA $(n=466)$} & \multirow[b]{2}{*}{$P$-value } & \multicolumn{2}{|c|}{ Stage IVB $(n=289)$} & \multirow[b]{2}{*}{$P$-value* } \\
\hline & $\begin{array}{l}\text { High FIB level } \\
\qquad(n=98)\end{array}$ & $\begin{array}{c}\text { Normal FIB level } \\
\quad(n=368)\end{array}$ & & $\begin{array}{l}\text { High FIB level } \\
\qquad(n=54)\end{array}$ & $\begin{array}{c}\text { Normal FIB level } \\
\qquad(n=235)\end{array}$ & \\
\hline 5y-DSS & $58.3 \%$ & $79.1 \%$ & $<0.001$ & $63.0 \%$ & $70.8 \%$ & 0.096 \\
\hline 5y-DFS & $57.1 \%$ & $72.6 \%$ & $<0.001$ & $53.7 \%$ & $64.7 \%$ & 0.081 \\
\hline 5y-DMFS & $62.3 \%$ & $87.5 \%$ & $<0.001$ & $53.6 \%$ & $82.0 \%$ & $<0.001$ \\
\hline
\end{tabular}

FIB = fibrinogen; High FIB level = FIB concentration $>4.0 \mathrm{~g} / \mathrm{L}$; Normal FIB level $=$ FIB concentration $\leq 4.0 \mathrm{~g} / \mathrm{L} ; \mathrm{DSS}=$ disease-specific survival; DFS = disease-free survival; DMFS = distant metastasis-free survival;; ${ }^{*} P$-values were calculated using the log-rank test.
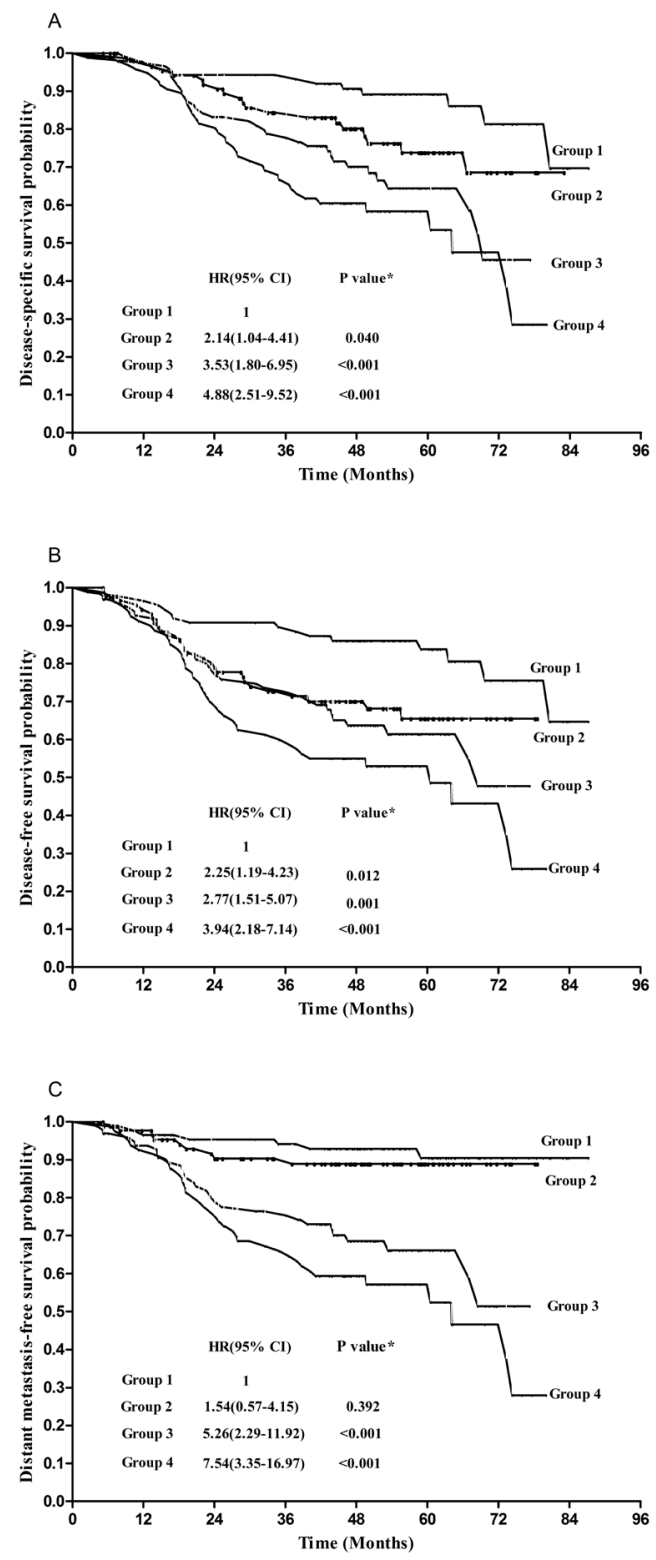

Figure 2: Kaplan-Meier survival curves to compare the disease-specific survival A., disease-free survival B., and distant metastasis-free survival C. for patients stratified by the pretreatment plasma FIB quartile values. Group 1: < 2.8g/L; Group 2: 2.8-3.6g/L; Group 3: 3.7-4.6g/L; Group 4: >4.6g/L. Hazards ratios (HR), 95\% confidence intervals (CIs) and $\mathrm{p}$ values were calculated using the unadjusted Cox proportional hazards model. 

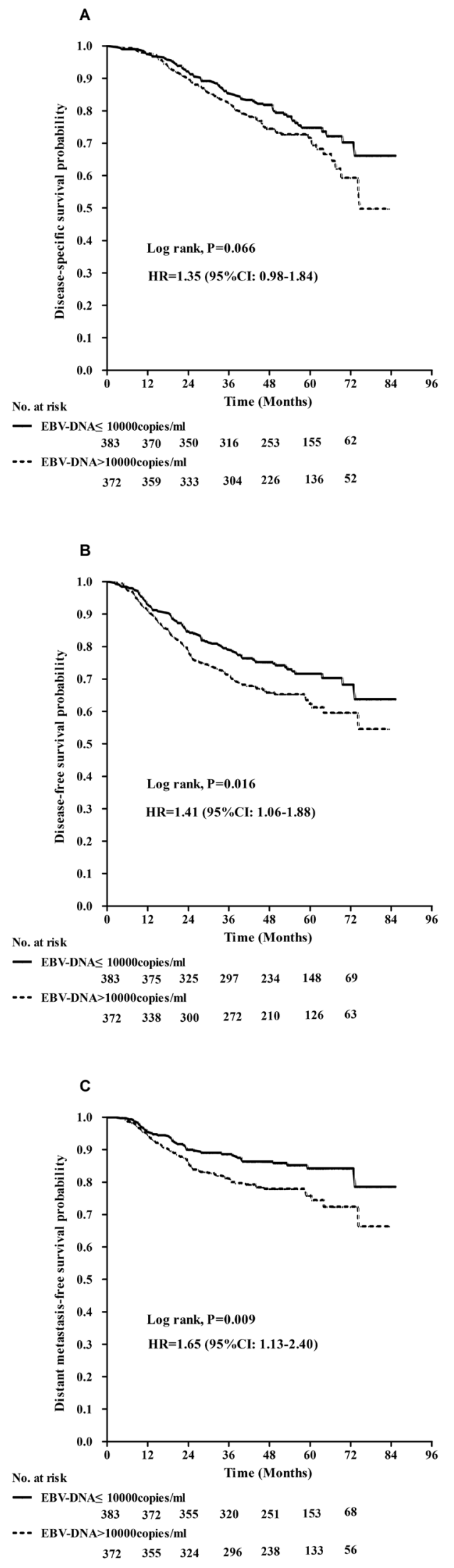

Figure 3: Kaplan-Meier curves show disease-specific survival A., disease-free survival B., and distant metastasis-free survival C. between patients with EBV-DNA $\leq 10000$ copies $/ \mathbf{m l}$ and EBV-DNA $>10000$ copies $/ \mathrm{ml}$. P values were calculated using an unadjusted log-rank test. 
( $>45$ years) and lactic acid dehydrogenase (LDH) were prognostic factors (Table 2). Multivariate analysis showed that the risk of distant metastasis in patients with high FIB level was over 3-fold than patients with normal FIB level. $\mathrm{LDH}>245 \mathrm{u} / \mathrm{L}$ was an independent negative prognostic factor for DSS and DFS; N stage was an independent prognostic factor for DMFS; and age $>45$ years was an independent prognostic factor for DSS. EBV-DNA was not an independent prognostic factor for any survival outcome in multivariate analysis (Table 3 ).

\section{DISCUSSION}

Our study demonstrates that elevated pretreatment plasma FIB is still associated with poor survival outcomes in stage IVA/B NPC in the era of IMRT; subgroup analysis confirmed DSS, DFS and DMFS decreased as FIB gradually increased, even within the normal range. Patients in our study had relatively high EBV-DNA values, with a median value of 10000 copies/ml (range, 0-10600000 copies/ml). Although EBV-DNA is established as one of the most effective prognostic factors in NPC, [15] it was not confirmed as an independent prognostic factor in this study of patients with stage IVA/B NPC treated with IMRT, indicating the prognostic value of EBV-DNA load should be reconsidered in this specific group of patients.

FIB is an acute phase glycoprotein traditionally associated with the maintenance of hemostasis that has been proven to be a significant prognostic factor in several solid tumor types. [6, 9, 10, 12-14, 19] Tang et al. [6] reported that increased EBV-DNA and fibrinogen levels alone and in combination are associated with reduced survival rates in stage I-IV NPC patients treated with either conventional radiotherapy, three-dimension conformal radiotherapy or IMRT. Our study reconfirmed that the gradual increases in FIB were associated with poorer survival outcomes in stage IVA/B diseases in the era of IMRT, but failed to confirm the value EBV-DNA load in this group of patients.

Independent prognostic factors, such as T category, clinical stage, gender, EBV-DNA load, established in other studies of locoregionally-advanced NPC (which always contain patients with both stage III and IVA/B diseases), $[6,20]$ did not show prognostic value in this cohort of patients with stage IVA/B diseases. Several factors may explain these differences. Firstly, with the widespread application of IMRT, prognostic factors for NPC may have changed over time. Secondly, patients with stage IVA/B disease are more likely to develop locoregional recurrence and distant metastasis than patients with stage III disease, and the factors that decrease the survival of patients with stage IVA/B are more complicated. Therefore, the prognostic factors identified for locoregionally-advanced NPC (both stage III and IVA/B) in previous studies may not be applicable to the specific group of patients with stage IVA/B diseases. In addition, with the development

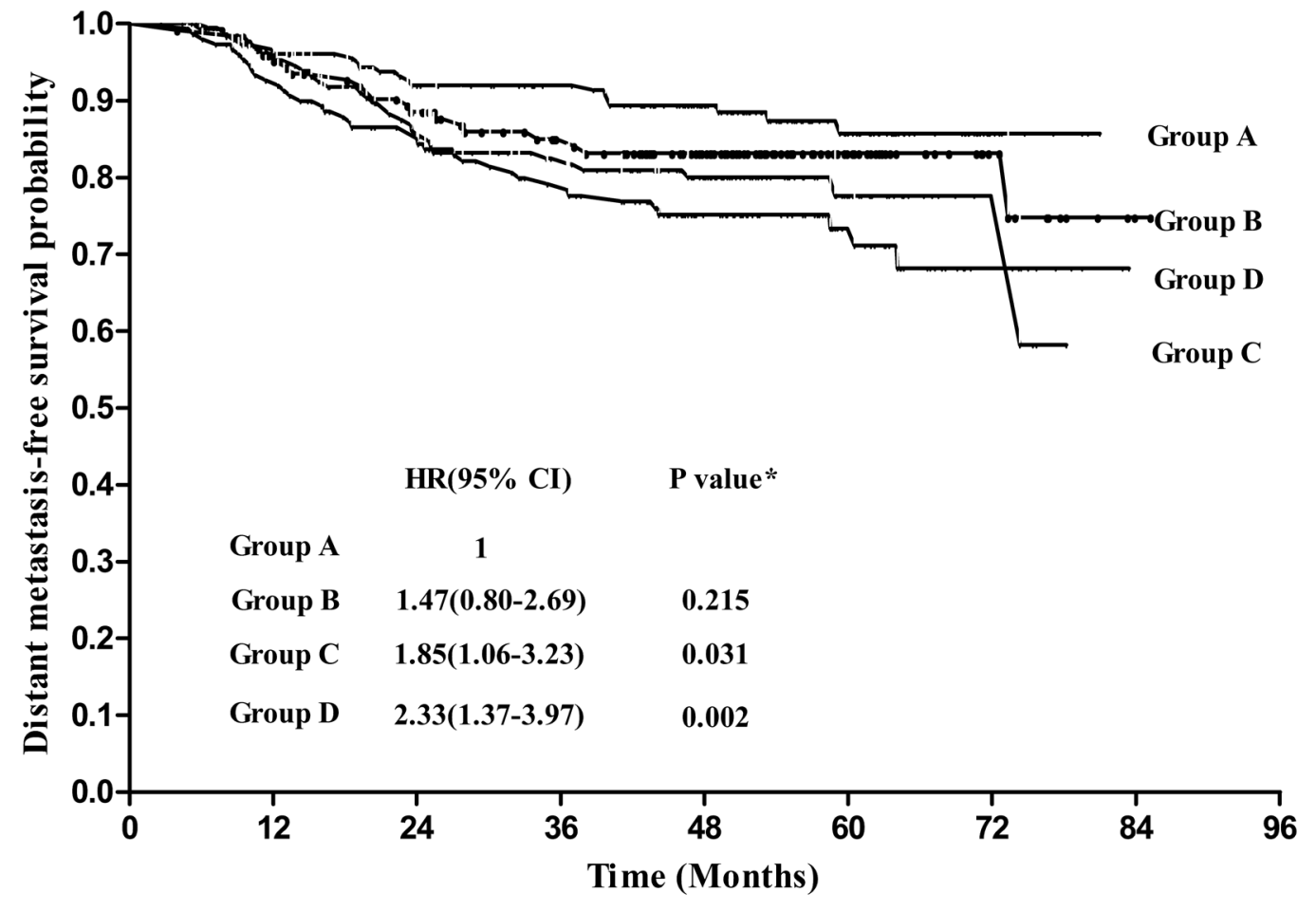

Figure 4: Distant metastasis-free survival for patients grouped according to pretreatment plasma EBV-DNA levels. Group A: <1000copies/ml; Group B: 1000-9999 copies/ml; Group C: 10000-50000copies/ml; Group D: >50000copies/ml. Hazards ratios (HR), 95\% confidence intervals (CIs) and p values were calculated using the unadjusted Cox proportional hazards model. 
Table 2: Univariate analysis of the associations between clinicopathological variables and clinical endpoints

\begin{tabular}{|c|c|c|c|c|c|c|}
\hline & 5-year DSS (\%) & $P$-value ${ }^{\dagger}$ & 5-year DFS (\%) & $P$-value ${ }^{\dagger}$ & $\begin{array}{c}\text { 5-year DMFS } \\
(\%)\end{array}$ & $P$-value ${ }^{\dagger}$ \\
\hline Age (years) & & 0.041 & & 0.193 & & 0.911 \\
\hline$\leq 45$ & 75.6 & & 68.0 & & 79.4 & \\
\hline$>45$ & 69.6 & & 65.6 & & 80.6 & \\
\hline Gender & & 0.111 & & 0.168 & & 0.264 \\
\hline Male & 71.1 & & 65.4 & & 79.2 & \\
\hline Female & 78.1 & & 71.3 & & 82.4 & \\
\hline T category & & 0.441 & & 0.645 & & 0.142 \\
\hline $\mathrm{T} 1$ & 64.2 & & 58.8 & & 69.7 & \\
\hline $\mathrm{T} 2$ & 80.7 & & 72.6 & & 86.8 & \\
\hline $\mathrm{T} 3$ & 72.0 & & 64.4 & & 76.5 & \\
\hline $\mathrm{T} 4$ & 72.1 & & 66.9 & & 80.3 & \\
\hline$N$ category & & 0.121 & & 0.101 & & 0.015 \\
\hline N0 & 79.6 & & 72.7 & & 86.3 & \\
\hline N1 & 75.1 & & 70.8 & & 85.0 & \\
\hline N2 & 72.6 & & 66.4 & & 77.1 & \\
\hline N3 & 69.2 & & 62.5 & & 76.0 & \\
\hline Clinical stage & & 0.042 & & 0.022 & & 0.009 \\
\hline IVa & 74.7 & & 69.3 & & 82.1 & \\
\hline $\mathrm{IVb}$ & 69.2 & & 62.5 & & 76.0 & \\
\hline FIB level & & $<0.001$ & & $<0.001$ & & $<0.001$ \\
\hline High level & 60.3 & & 56.0 & & 59.4 & \\
\hline Normal level & 76.0 & & 69.9 & & 85.5 & \\
\hline $\begin{array}{l}\text { EBV-DNA } \\
\text { (copies/ml) }\end{array}$ & & 0.066 & & 0.016 & & 0.009 \\
\hline$>10000$ & 71.7 & & 62.0 & & 75.8 & \\
\hline$\leq 10000$ & 74.8 & & 71.4 & & 84.2 & \\
\hline LDH (U/L) & & 0.002 & & 0.001 & & $<0.001$ \\
\hline$>245$ & 61.8 & & 53.7 & & 66.2 & \\
\hline$\leq 245$ & 74.9 & & 68.5 & & 81.8 & \\
\hline
\end{tabular}

DSS = disease-specific survival; DFS = disease-free survival; DMFS = distant metastasis-free survival; FIB = fibrinogen; High FIB level $=$ FIB concentration $>4.0 \mathrm{~g} / \mathrm{L}$; Normal FIB level $=$ FIB concentration $\leq 4.0 \mathrm{~g} / \mathrm{L}$; EBV-DNA = Epstein-Barr virus DNA; LDH = lactic acid dehydrogenase; $\uparrow P$-values were calculated using the unjusted log-rank test.

of conformal radiotherapy technology, such as IMRT, and the use of chemotherapy, the 5-year overall survival rate for stage III NPC is reported to have reached almost $90 \%$, but remains around $60 \%-70 \%$ for stage IVA/B disease. [3, $21,22]$ To some extent, these survival differences may reflect inherent biological variations that affect the value of such prognostic factors in patients with stage III and stage IVA/B NPC.

The mechanisms by which FIB affects the survival of patients with cancer have been intensively researched; the evidence indicates that FIB plays a key role in both inflammation and cancer progression. [23] However, the precise molecular mechanisms underlying the relationship 
Table 3: Summary of multivariate analysis of the associations between clinicopathological variables and clinical endpoints

\begin{tabular}{|c|c|c|c|c|c|c|}
\hline \multirow[t]{2}{*}{ Characteristic } & \multicolumn{2}{|c|}{$5 y-D S S$} & \multicolumn{2}{|c|}{ 5y-DFS } & \multicolumn{2}{|c|}{ 5y-DMFS } \\
\hline & HR $(95 \% \mathrm{CI})^{\dagger}$ & $P$-value ${ }^{*}$ & HR $(95 \% \mathrm{CI})^{\dagger}$ & $P$-value & HR $(95 \% \mathrm{CI})^{\dagger}$ & $P$-value \\
\hline High FIB level & $1.94(1.41-2.68)$ & $<0.001$ & $1.51(1.09-2.10)$ & 0.013 & $3.17(2.14-4.69)$ & $<0.001$ \\
\hline $\mathrm{LDH}>245 \mathrm{U} / \mathrm{L}$ & $1.59(1.06-2.39)$ & 0.026 & $1.60(1.07-2.39)$ & 0.022 & $1.57(0.95-2.58)$ & 0.076 \\
\hline Age $>45$ years & $1.37(1.02-1.83)$ & 0.036 & & & & \\
\hline $\mathrm{N}$ category & & & & & $1.53(1.00-2.35)$ & 0.049 \\
\hline EBV-DNA>10000copies $/ \mathrm{ml}$ & & & $1.26(0.94-1.70)$ & 0.125 & $1.26(0.92-1.65)$ & 0.128 \\
\hline
\end{tabular}

DSS = disease-specific survival; DFS = disease-free survival; DMFS = distant metastasis-free survival; FIB = fibrinogen; High FIB level $=$ FIB concentration $>4.0 \mathrm{~g} / \mathrm{L} ;$ Normal FIB level $=$ FIB concentration $\leq 4.0 \mathrm{~g} / \mathrm{L} ; \mathrm{LDH}=$ lactic acid dehydrogenase; $\dagger$ Hazard ratios; * $P$-values were calculated using the Cox proportional hazards model.

between high pretreatment plasma FIB and poorer survival in patients with solid tumors have not been fully clarified.

Consistent with results of previous studies [24, 25], elevated LDH was confirmed as a prognostic factor for poorer survival in this cohort of patients with stage IVA/B NPC, further indicating that high LDH may be associated with a larger tumor burden, tumor extension and higher risk of metastasis.

One major limitation of this study is that only pretreatment plasma FIB was evaluated; we did not evaluate the changes in FIB during and after treatment. As most patients underwent several cycles of chemotherapy, all patients received peripherally-inserted central catheters and/or subclavian vein catheters, which could induce coagulation and activation of fibrinolysis. Secondly, this was a retrospective study of patients treated at a single institute; therefore, these results need to be validated in data sets from other institutions and in prospective studies. Furthermore, the median follow-up period was relatively short (50 months); therefore, long-term validation is required.

In conclusion, high pretreatment FIB level is associated with poorer DSS, DFS and DMFS, and presents to be a better prognostic factor than EBV-DNA for patients with stage IVA/B NPC in the IMRT era.

\section{MATERIALS AND METHODS}

\section{Patients and work-up}

This retrospective study was approved by the institutional review board. In total, 755 patients with newly-diagnosed stage IVA/B NPC who were treated with definitive IMRT, between January 2007 and December 2011, were analyzed in this study. Patients were restaged according to the American Joint Committee on Cancer/ International Union Against Cancer (AJCC/UICC) 2010 staging system. Patients with concomitant disease suspected of influencing plasma FIB, such as severe hypertension, liver disease or blood coagulation disorders, were excluded.

All patients underwent a pretreatment evaluation which consisted of a complete physical examination, hematologic and biochemistry profiles, magnetic resonance imaging (MRI) of the nasopharynx and neck, chest radiography or computed tomography (CT), abdominal ultrasonography, and emission CT or wholebody positron emission tomography/CT. Plasma EBVDNA and FIB were measured for all patients before treatment.

A higher number of patients had stage IVA (466/755; $61.7 \%)$ than stage IVB disease $(289 / 755 ; 38.3 \%)$. The male $(n=578) /$ female $(n=177)$ ratio was $3.3: 1$ and the median age was 45 years (range, 11-78 years). Pathologically, only 3/755 (0.4\%) patients had World Health Organization (WHO) type I disease; the remainder had type II or type III disease (Table 4).

\section{Measurement of fibrinogen and EBV-DNA}

Plasma FIB and EBV-DNA are routinely assessed before treatment in our hospital. A $3 \mathrm{~mL}$ blood sample was collected and coagulation function was automatically assessed within $3 \mathrm{~h}$ of collection using an Analyzer Sysmex CA7000 (Sysmex Corporation, Kobe, Japan) and reagents in accordance with the SIEMENS AG guidelines (Munich, Germany) (Clauss, 1957; Cook and Ubben, 1990) [6]. According to the instructions, a plasma FIB concentration $\leq 4.0 \mathrm{~g} / \mathrm{L}$ was considered normal, and $>4.0$ $\mathrm{g} / \mathrm{L}$ was defined as hyperfibrinogenemia or high. Using this cut-off value, the patients were classified into the normal FIB and high FIB groups.

Plasma EBV-DNA load was routinely measured by real-time quantitative PCR (RT-qPCR) before treatment using a method that has previously been described in detail. $[15,18]$ 


\begin{tabular}{|c|c|}
\hline Characteristic & Number of patients (\%) \\
\hline \multicolumn{2}{|l|}{ Age (years) } \\
\hline$\leq 45$ & $371(49.1)$ \\
\hline$>45$ & $384(50.9)$ \\
\hline \multicolumn{2}{|l|}{ Gender } \\
\hline Male & $578(76.6)$ \\
\hline Female & $177(23.4)$ \\
\hline \multicolumn{2}{|l|}{ WHO type } \\
\hline $\mathrm{III}+\mathrm{II}$ & $752(99.6)$ \\
\hline I & $3(0.4)$ \\
\hline \multicolumn{2}{|l|}{ T category } \\
\hline $\mathrm{T} 1$ & $17(2.3)$ \\
\hline $\mathrm{T} 2$ & $59(7.8)$ \\
\hline $\mathrm{T} 3$ & $147(19.5)$ \\
\hline $\mathrm{T} 4$ & $532(70.4)$ \\
\hline \multicolumn{2}{|l|}{$\mathrm{N}$ category } \\
\hline No & $53(7.0)$ \\
\hline N1 & $232(30.7)$ \\
\hline $\mathrm{N} 2$ & $181(24.0)$ \\
\hline N3 & $289(38.3)$ \\
\hline \multicolumn{2}{|l|}{ Clinical stage } \\
\hline IVA & $466(61.7)$ \\
\hline IVB & $289(38.3)$ \\
\hline \multicolumn{2}{|l|}{ FIB level } \\
\hline High FIB level & $152(20.1)$ \\
\hline Normal FIB level & $603(79.9)$ \\
\hline
\end{tabular}

Abbreviations: $\mathrm{WHO}=$ World Health Organization; FIB $=$ fibrinogen.

\section{Treatment}

All patients received definitive IMRT that covered the nasopharynx and retropharyngeal lymph nodes within the primary target volumes. Whole-neck irradiation was performed in all cases. The details of the radiotherapy technique used at our cancer center have previously been reported. $[3,20]$ Most patients $(723 / 755 ; 95.8 \%)$ received platinum-based chemotherapy: neoadjuvant (546/755; $72.3 \%)$, concurrent $(548 / 755 ; 72.5 \%)$ and adjuvant chemotherapy $(33 / 755 ; 4.4 \%)$.

\section{Follow-up and statistical analysis}

Follow-up was measured from the day of diagnosis to the date of the event or last follow-up visit. All patients were followed up by phone calls for the first 3 months, every 3 months for the next 3 years, every 6 months for the next 2 years, and then annually. Disease-specific survival (DSS), disease-free survival (DFS) and distant metastasis-free survival (DMFS) were calculated using the Kaplan-Meier method, and differences were compared using the log-rank test. DSS was defined as the time interval from diagnosis to death caused by cancer or censorship at last follow-up; DFS, to first observation of progression, death or censorship at last follow-up; and DMFS, to first observation of distant lesions or censorship at last follow-up.

For subgroup analysis, the patients were divided into four groups based on the FIB quartile values. Univariate analysis was conducted using the log-rank test. Variables with $P$-values $<0.05$ in univariate analysis 
were included in the final multivariate survival analysis, which was conducted using the Cox proportional hazards model. Statistical Package for the Social Sciences (SPSS, Chicago, IL, USA), version 20.0 was used for all analyses. Two-tailed $P$-values $<0.05$ were considered statistically significant.

\section{ACKNOWLEDGMENTS}

We would like to thank professor Qing Liu (Department of Cancer Prevention Research, Sun YatSen University Cancer Center) for providing us with suggestions on statistical analysis.

\section{CONFLICTS OF INTEREST}

All the authors of this paper declare that they have no conflicts of interest.

\section{GRANT SUPPORT}

This work was supported by Grants from the Natural Science Foundation of China (81402244).

\section{REFERENCES}

1. Yau TK, Lee AW, Wong DH, Pang ES, Ng WT, Yeung RM, Soong IS. Treatment of Stage IV(A-B) nasopharyngeal carcinoma by induction-concurrent chemoradiotherapy and accelerated fractionation: impact of chemotherapy schemes. International journal of radiation oncology, biology, physics. 2006; 66:1004-1010.

2. Hong RL, Ting LL, Ko JY, Hsu MM, Sheen TS, Lou PJ, Wang CC, Chung NN, Lui LT. Induction chemotherapy with mitomycin, epirubicin, cisplatin, fluorouracil, and leucovorin followed by radiotherapy in the treatment of locoregionally advanced nasopharyngeal carcinoma. Journal of clinical oncology. 2001; 19:4305-4313.

3. Zeng L, Tian YM, Sun XM, Huang Y, Chen CY, Han F, Liu S, Lan M, Guan Y, Deng XW, Lu TX. Intensity-modulated radiotherapy for stage IVA/IVB nasopharyngeal carcinoma: clinical outcomes and patterns of failure in an endemic area in China. Strahlenther Onkol. 2014; 190:993-1000.

4. Coussens LM, Werb Z. Inflammation and cancer. Nature. 2002; 420:860-867.

5. Bardos H, Molnar P, Csecsei G, Adany R. Fibrin deposition in primary and metastatic human brain tumours. Blood coagulation \& fibrinolysis. 1996; 7:536-548.

6. Tang LQ, Chen QY, Guo SS, Chen WH, Li CF, Zhang L, Lai XP, He Y, Xu YX, Hu DP, Wen SH, Peng YT, Liu H, Liu LT, Yan SM, Guo L, et al. The impact of plasma Epstein-Barr virus DNA and fibrinogen on nasopharyngeal carcinoma prognosis: an observational study. Br J Cancer. 2014; 111:1102-1111.
7. Palumbo JS, Talmage KE, Massari JV, La Jeunesse CM, Flick MJ, Kombrinck KW, Jirouskova M, Degen JL. Platelets and fibrin(ogen) increase metastatic potential by impeding natural killer cell-mediated elimination of tumor cells. Blood. 2005; 105:178-185.

8. Simpson-Haidaris PJ, Rybarczyk B. Tumors and fibrinogen. The role of fibrinogen as an extracellular matrix protein. Annals of the New York Academy of Sciences. 2001; 936:406-425.

9. Perisanidis C, Psyrri A, Cohen EE, Engelmann J, Heinze G, Perisanidis B, Stift A, Filipits M, Kornek G, Nkenke E. Prognostic role of pretreatment plasma fibrinogen in patients with solid tumors: A systematic review and metaanalysis. Cancer treatment reviews. 2015.

10. Palumbo JS, Kombrinck KW, Drew AF, Grimes TS, Kiser JH, Degen JL, Bugge TH. Fibrinogen is an important determinant of the metastatic potential of circulating tumor cells. Blood. 2000; 96:3302-3309.

11. Zhang D, Zhou X, Bao W, Chen Y, Cheng L, Qiu G, Sheng L, Ji Y, Du X. Plasma fibrinogen levels are correlated with postoperative distant metastasis and prognosis in esophageal squamous cell carcinoma. Oncotarget. 2015; 6:38410-38420. doi: 10.18632/oncotarget.4800.

12. Wen J, Yang Y, Ye F, Huang X, Li S, Wang Q, Xie X. The preoperative plasma fibrinogen level is an independent prognostic factor for overall survival of breast cancer patients who underwent surgical treatment. Breast (Edinburgh, Scotland). 2015; 24:745-750.

13. Pavey SJ, Hawson GA, Marsh NA. Impact of the fibrinolytic enzyme system on prognosis and survival associated with non-small cell lung carcinoma. Blood coagulation \& fibrinolysis. 2001; 12:51-58.

14. Jagielska B, Symonides M, Stachurska E, Kawecki A, Kraszewska E. Coagulation disorders in patients with locally advanced head and neck cancer - should they really be disregarded? Neoplasma. 2011; 58:129-134.

15. Shao JY, Li YH, Gao HY, Wu QL, Cui NJ, Zhang L, Cheng $\mathrm{G}, \mathrm{Hu} \mathrm{LF}$, Ernberg I, Zeng YX. Comparison of plasma Epstein-Barr virus (EBV) DNA levels and serum EBV immunoglobulin A/virus capsid antigen antibody titers in patients with nasopharyngeal carcinoma. Cancer. 2004; 100:1162-1170.

16. Liu LT, Tang LQ, Chen QY, Zhang L, Guo SS, Guo L, Mo HY, Zhao C, Guo X, Cao KJ, Qian CN, Zeng MS, Bei JX, Hong MH, Shao JY, Sun Y, et al. The Prognostic Value of Plasma Epstein-Barr Viral DNA and Tumor Response to Neoadjuvant Chemotherapy in AdvancedStage Nasopharyngeal Carcinoma. International journal of radiation oncology, biology, physics. 2015; 93:862-869.

17. Wang WY, Twu CW, Chen HH, Jan JS, Jiang RS, Chao JY, Liang KL, Chen KW, Wu CT, Lin JC. Plasma EBV DNA clearance rate as a novel prognostic marker for metastatic/ recurrent nasopharyngeal carcinoma. Clinical cancer research. 2010; 16:1016-1024. 
18. An X, Wang FH, Ding PR, Deng L, Jiang WQ, Zhang L, Shao JY, Li YH. Plasma Epstein-Barr virus DNA level strongly predicts survival in metastatic/recurrent nasopharyngeal carcinoma treated with palliative chemotherapy. Cancer. 2011; 117:3750-3757.

19. Palumbo JS, Degen JL. Fibrinogen and tumor cell metastasis. Haemostasis. 2001; 31:11-15.

20. Sun X, Su S, Chen C, Han F, Zhao C, Xiao W, Deng $\mathrm{X}$, Huang S, Lin C, Lu T. Long-term outcomes of intensity-modulated radiotherapy for 868 patients with nasopharyngeal carcinoma: an analysis of survival and treatment toxicities. Radiotherapy and oncology. 2014; 110:398-403.

21. Lee AWM, Sze WM, Au JSK, Leung SF, Leung TW, Chua DTT, Zee BCY, Law SCK, Teo PML, Tung SY, Kwong DLW, Lau WH. Treatment results for nasopharyngeal carcinoma in the modern era: The Hong Kong experience. International Journal of Radiation Oncology Biology Physics. 2005; 61:1107-1116.

22. Lan M, Huang Y, Chen CY, Han F, Wu SX, Tian L, Zheng L, Lu TX. Prognostic Value of Cervical Nodal Necrosis in Nasopharyngeal Carcinoma: Analysis of 1800 Patients with Positive Cervical Nodal Metastasis at MR Imaging. Radiology. 2015; 276:536-544.

23. Palumbo JS, Degen JL. Mechanisms coupling the hemostatic system to colitis-associated cancer. Thrombosis research. 2010; 125:S39-S43.

24. Wan XB, Wei L, Li H, Dong M, Lin Q, Ma XK, Huang PY, Wen JY, Li X, Chen J, Ruan DY, Lin ZX, Chen ZH, Liu Q, Wu XY, Hong MH. High pretreatment serum lactate dehydrogenase level correlates with disease relapse and predicts an inferior outcome in locally advanced nasopharyngeal carcinoma. European journal of cancer (Oxford, England : 1990). 2013; 49:2356-2364.

25. Jin Y, Ye X, Shao L, Lin BC, He CX, Zhang BB, Zhang YP. Serum lactic dehydrogenase strongly predicts survival in metastatic nasopharyngeal carcinoma treated with palliative chemotherapy. European journal of cancer (Oxford, England : 1990). 2013; 49:1619-1626. 